Authors' reply: Smith et al worry that we have underestimated the role of genetics in early-onset depression. They draw our attention to the published evidence for the importance of genetic factors in prepubertal depression, which itself is a marker for adult bipolar disorder, although not necessarily a marker for major depression (the topic of our editorial). From their own data, they report that three-quarters of 47 subjects who had a depressive episode by a mean age of 15 had a first-degree relative with an affective disorder. Strong evidence indeed of familiarity, but not necessarily of a genetic cause. Despite their certainty, many of us have problems with the precise nature of the evidence supporting genetic factors in major depression, in part because of the dimensional nature of depression, and in part because of the extensive comorbidity.

We opined that the heightened risk of depression in young people whose parents had depression was likely to be 'more psychological than genetic' and referred the reader to the review by Beardslee et al (1998). We provided evidence that intervention programmes for adolescents can reduce by half the probability of depression in the future. Smith et al argue that universal interventions are unlikely to be effective until we have complete understanding of how genetic and non-genetic factors interact to bring about the depressive phenotype, and that interventions targeted to high-risk groups should be deferred until we can reliably identify those individuals at high risk.

We strongly oppose this thinking. Most interventions in medicine are introduced before there is a complete understanding of the aetiology of the disorder, and usually before there is precise information as to which individuals will respond. Simply to know that an intervention can produce a reliable and significant benefit is sufficient to warrant implementation. We believe that this is the situation in regard to the prevention of major depression in young people.

Beardslee, W. R., Versage, E. M. \& Gladstone, T. R. G. (1998) Children of affectively ill parents: a review of the past 10 years. Journal of the American Academy of Child and Adolescent Psychiatry, 37, ||34-||4|.

G. Andrews, M. Szabo, J. Burns School of Psychiatry, The University of New South Wales, Sydney 2052, Australia

\section{Depressive symptoms and cognitive decline}

In their recent paper, Paterniti et al (2002) reported that depressive symptoms predict cognitive decline over a 4-year period. This is a well-designed and well-written study that replicates a previous finding from similarly well-designed studies. Negative findings on this question, however, are also common in the literature, including a report from the same French group a few years ago (Dufouil et al, 1996). I would like to point out some relevant issues overlooked by Paterniti et al.

First, I find it unfortunate that the paper cites few negative reports, with no mention in the discussion of the many longitudinal studies that have reported no association between depressive states and subsequent cognitive decline (Dufouil et al, 1996; Prince et al, 1996; Cervilla $e t$ al, 2000). It is particularly surprising that Paterniti et al quote the study by Chen et al (1999) as reporting that 'depressive symptoms are predictive of cognitive decline', when in fact they found that dementia predicted the onset of depressive symptoms but not the other way round.

Second, it is regrettable that Paterniti et al overlooked the only study to date addressing the very same question but for a considerably longer follow-up period (Cervilla et al, 2000). Longer follow-up periods could help to distinguish between psychopathology shared by depression and dementia (e.g. difficulties with memory and concentration, or apathy), as pointed out by a previous study by some co-authors of Paterniti's paper (Dufouil et al, 1996). Indeed it could be argued that if depressive symptoms have a real capacity to predict cognitive decline, the latter should be expected to become more apparent as the study's follow-up period lengthens. This, in fact, has not happened in our cohort (Prince et al, 1996; Cervilla et al, 2000) and I believe this adds potentially unique information to Paterniti et al's discussion.

Finally, it is also regrettable that Paterniti's group did not explore the repeatedly reported interaction with gender in considering whether depressive symptoms predict cognitive decline (Prince et al, 1996; Cervilla et al, 2000). Indeed, the latter studies have reported that if an association exists between depressive symptoms and cognitive decline, this seems to be the case in men only (Cervilla et al, 2000), or in men of above-median premorbid IQ (Prince et al, 1996).

Cervilla, J., Prince, M, Joels, S., et al (2000) Does depression predict cognitive outcome 9 to 12 years later? Evidence from a prospective study of elderly hypertensives. Psychological Medicine, 30, 1017-1023.

Chen, T., Ganguly, M., Mulsant, B., et al (1999) The temporal relationship between depressive symptoms and dementia. A community prospective study. Archives of General Psychiatry, 56, 25I-266.

Dufouil, C., Fuhrer, R., Dartigues, J. F., et al (1996) Longitudinal analysis of the association between depressive symptomatology and cognitive deterioration. American Journal of Epidemiology, 144, 634-641.

Paterniti, S., Verdier-Taillefer, M. H., Dufouil, C., et al (2002) Depressive symptoms and cognitive decline in elderly people. Longitudinal study. British Journal of Psychiatry, I8I, 406-410.

Prince, M. J., Lewis, G., Bird, A., et al (1996) A longitudinal study of factors predicting change in cognitive test scores over time, in an older hypertensive population. Psychological Medicine, 26, 555-568.

J. Cervilla Sant Joan de Deu - SSM, Vilanova i la Geltrú, Barcelona, Spain

\section{Preventing suicide}

In his editorial, De Leo (2002) cites important papers of the past 8 years. He does not mention that over 100 years ago the great sociologist, Emile Durkheim (1897), stated that the suicide rate reflected patterns of social relationships within communities and that individual mental disorder had little bearing on this behaviour. His view has never been effectively refuted.

De Leo does, however, observe that 'socio-economic events' such as wars and economic fluctuations may 'provoke effects' that 'would be incomparably bigger than any well-targeted anti-suicide initiative'. He recognised that in most Western countries, there is currently a 'remarkable decline' in youth suicide, which cannot be attributed to suicide prevention activities. Over the past 50 years, there have been synchronous, international trends in suicide (La Vecchia et al, 1994). All of these events are probably due to sociocultural influences rather than fluctuations in the prevalence of mental disorders, and substantiate Durkheim's view.

De Leo states that suicidal behaviour attracts little interest among contemporary psychiatrists, as judged by the low number of contributions to suicidology journals. But this would seem to be the wrong yardstick. If Durkheim's view is accepted, the most profitable approach to the prevention of suicide would be the creation of full employment and supportive environments, and the reduction of family breakdown 